\title{
Embedded Real-Time Model Predictive Control for Glucose Regulation
}

\author{
Chee-Kong Chui ${ }^{1}$, Binh P. Nguyen ${ }^{1}$, Yvonne Ho ${ }^{1}$, Zimei Wu ${ }^{1}$, Mai Nguyen ${ }^{1}$, Geok-Soon Hong ${ }^{1}$, Daniel \\ $\mathrm{Mok}^{2}$, Sumei Sun ${ }^{2}$ and Stephen Chang ${ }^{3}$ \\ ${ }^{1}$ Department of Mechanical Engineering, National University of Singapore, Singapore 117576 \\ ${ }^{2}$ Institute for Infocomm Research, A*STAR, Singapore 138632 \\ ${ }^{3}$ Department of Surgery, National University Hospital, Singapore 119074
}

\begin{abstract}
This paper reports our investigation on a model predictive control (MPC) with constraints for continuous diabetes management, and its implementation on the microcontroller of our artificial pancreas. The operational constraints for the MPC are rate of change, amplitude and output constraints, while the associated optimization problem is solved using a primal-dual interior-point algorithm based on predicatorcorrector method. Our real-time implantable close-loop system is able to achieve desired diabetes management by maintaining the blood sugar level at less than $140 \mathrm{mg} / \mathrm{dl}$, and consistently within the range of 70-120 mg/dl. Experimental results demonstrate that the low power 16-bit microcontroller in our prototype artificial pancreas can provide sufficient computational power with our computational efficient embedded system solution.
\end{abstract}

Keywords - Model predictive control, artificial pancreas, minimal model, embedded system.

\section{INTRODUCTION}

Diabetes Mellitus is a widespread disease caused by high blood glucose in the patient's body (Diabetes.com). High blood sugar sets off processes that can lead to complications, like heart, kidney, and eye disease, or other serious problems. There are two types of Diabetes: Type 1 and type 2 Diabetes. In addition to medication and/or insulin shots, Type 1 patients and some Type 2 patients are required to measure their blood sugar level every day with the aid of a blood glucose monitor. A blood glucose monitor or glucose meter is a small portable battery-powered electronic device mainly used to determine the deviations of the patient's blood glucose level from the normal healthy level. There are many different types of blood glucose monitors in the market for diabetes management. $\mathrm{Pa}$ tients may be required to use lancets to draw blood from their finger. Recent models allow people to test the glucose level with blood extracted from other areas. The most recent models use sensor pod that is placed underneath the skin to constantly measure the blood sugar via an external monitoring device. Sensors from the latest model could be used continuously for up to a week. There are many interests in the health- care industry to develop an implantable solution or artificial pancreas that could remove the patient's pains from blood extraction and needle insertion as well as better diabetes management outcomes. To provide a real-time close-loop blood glucose regulation system for diabetes management in artificial pancreas is a challenge [1]. This paper reports our investigation on a model predictive control (MPC) with constraints for continuous diabetes management, and its implementation on a microcontroller for our artificial pancreas prototype.

\section{Model Predictive CONTROL}

Assuming the plant has $m$ inputs, $q$ outputs, and $n_{1}$ states, the formulation of the predictive control problem can be stated as below:

$$
\begin{aligned}
x_{m}(k+1) & =A_{m} x_{m}(k)+B_{m} u(k), \\
y(k) & =C_{m} x_{m}(k),
\end{aligned}
$$

where $u$ is the manipulated or input variable, $y$ is the process output, and $x_{m}$ is the state variable; $A_{m}, B_{m}$, and $C_{m}$ have dimensions of $n_{1} \times n_{1}, n_{1} \times m$, and $q \times n_{1}$, respectively.

Denoting $\Delta x_{m}(k)=x_{m}(k)-x_{m}(k-1), \Delta u(k)=u(k)-$ $u(k-1)$, and $x(k)=\left[\begin{array}{ll}\Delta x_{m}(k)^{T} & y(k)\end{array}\right]^{T}$, with a difference operation on both sides of (1a) and (1b), we obtain the following state-space model:

$$
\begin{gathered}
\underbrace{\left[\begin{array}{c}
\Delta x_{m}(k+1) \\
y(k+1)
\end{array}\right]}_{x(k+1)}=\underbrace{\left[\begin{array}{cc}
A_{m} & o_{m}^{T} \\
C_{m} A_{m} & I_{q \times q}
\end{array}\right]}_{A} \underbrace{\left[\begin{array}{c}
\Delta x_{m}(k) \\
y(k)
\end{array}\right]}_{x(k)}+\underbrace{\left[\begin{array}{c}
B_{m} \\
C_{m} B_{m}
\end{array}\right]}_{B} \Delta u(k), \\
y(k)=\underbrace{\left[\begin{array}{cc}
o_{m} & I_{q \times q}
\end{array}\right]}_{C} \underbrace{\left[\begin{array}{c}
\Delta x_{m}(k) \\
y(k)
\end{array}\right]}_{x(k)},
\end{gathered}
$$

where $o_{m}$ is a $q \times n_{1}$ zero matrix, and $I_{q \times q}$ is the identity matrix with dimensions of $q \times q$.

At the sampling instant $k_{i}$, we assume that the state variable $x\left(k_{i}\right)$ is available to provide the current information of the plant. Then, the future state variables are predicted for $N_{p}$ samples: $x\left(k_{i}+1 \mid k_{i}\right), x\left(k_{i}+2 \mid k_{i}\right), \ldots, x\left(k_{i}+N_{p} \mid k_{i}\right)$, and the optimal future control trajectory is described within $N_{c}$ samples 
$\left(N_{c} \leq N_{p}\right): \Delta u\left(k_{i}\right), \Delta u\left(k_{i}+1\right), \ldots, \Delta u\left(k_{i}+N_{c}-1\right)$. By defining

$$
\Delta U=\left[\Delta u\left(k_{i}\right)^{T} \Delta u\left(k_{i}+1\right)^{T} \cdots \Delta u\left(k_{i}+N_{c}-1\right)^{T}\right]^{T},
$$$$
Y=\left[y\left(k_{i}+1 \mid k_{i}\right)^{T} y\left(k_{i}+2 \mid k_{i}\right)^{T} \cdots y\left(k_{i}+N_{p} \mid k_{i}\right)^{T}\right]^{T} \text {, }
$$

and sequentially calculating the predicted future state and output variables using the future control parameters, we obtain

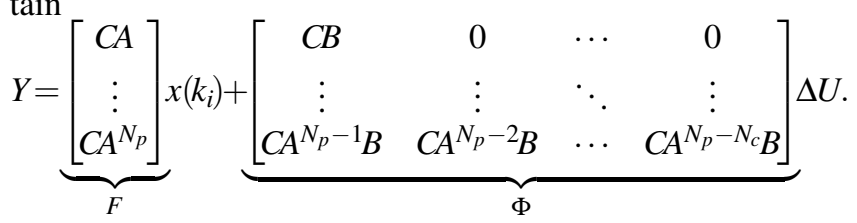

For a given set-point signal $r\left(k_{i}\right)=$ $\left[\begin{array}{llll}r_{1}\left(k_{i}\right) & r_{2}\left(k_{i}\right) & \cdots & r_{q}\left(k_{i}\right)\end{array}\right]^{T}$ at sample time $k_{i}$, we need to find the optimal control parameter vector $\Delta U$ so that within the prediction horizon, the predicted output variables are as close as possible to the set-points. The cost function that reflects this control objective is defined as

$$
J=\left(R_{s}-Y\right)^{T}\left(R_{s}-Y\right)+\Delta U^{T} \bar{R} \Delta U,
$$

where $R_{s}=\left[\begin{array}{llll}I_{q \times q} & I_{q \times q} & \cdots & I_{q \times q}\end{array}\right]^{T} r\left(k_{i}\right)=\bar{R}_{s} r\left(k_{i}\right)$, and $\bar{R}$ is a block matrix with $m \times m$ blocks of $r_{\omega} I_{N_{c} \times N_{c}}\left(r_{\omega} \geq 0\right)$, in which $r_{\omega}$ is a tuning parameter, and $I_{N_{c} \times N_{c}}$ is the $N_{c} \times N_{c}$ identity matrix.

From (4) and (5), by solving $\frac{\partial J}{\partial \Delta U}=0$ for the minimum of $J$, the optimal solution is:

$$
\Delta U=\left(\Phi^{T} \Phi+\bar{R}\right)^{-1} \Phi^{T}\left(R_{s}-F x\left(k_{i}\right)\right)
$$

Note that only the first $m$ elements of $\Delta U, \Delta u\left(k_{i}\right)$, will be utilized. At next sample time, the new state variable $x\left(k_{i}+1\right)$ is measured and used to calculate the new control trajectory.

At time $k_{i}$, the state variable $x\left(k_{i}\right)$ is assumed to be available. However, in practice, not all state variables are available or measurable. A solution to this issue is to estimate the state variable $x(k)$ using a state observer. We calculate the state variable $\hat{x}_{m}(k)$ based on the plant model in (1), and use the error signal of output as a feedback to improve the estimation as below

$$
\hat{x}_{m}(k+1)=A_{m} \hat{x}_{m}(k)+B_{m} u(k)+K_{o b}\left(y(k)-C_{m} \hat{x}_{m}(k)\right),
$$

where $K_{o b}$ is the observer gain matrix.

By substituting (1b) into (7), we deduce that the error $\tilde{x}_{m}(k)=x_{m}(k)-\hat{x}_{m}(k)$ satisfies the difference equation: $\tilde{x}_{m}(k+1)=A_{m} \tilde{x}_{m}(k)-K_{o b} C_{m} \tilde{x}_{m}(k)=\left(A_{m}-K_{o b} C_{m}\right) \tilde{x}_{m}(k)$.

For a given initial error state $\tilde{x}_{m}(0) \neq 0$, we have

$$
\tilde{x}_{m}(k)=\left(A_{m}-K_{o b} C_{m}\right)^{k} \tilde{x}_{m}(0) \text {. }
$$

If the observer gain $K_{o b}$ is chosen so that the error system matrix $A_{m}-K_{o b} C_{m}$ has all eigenvalues inside the unit circle, then the error system (8) is stable and $\left\|\tilde{x}_{m}(k)\right\| \rightarrow 0$ as $k \rightarrow \infty$. This means that the estimated state variable $\hat{x}_{m}(k)$ converges to $x_{m}(k)$.
At sample time $k_{i}$, with the information of $\hat{x}\left(k_{i}\right)$ replacing $x\left(k_{i}\right)$, the estimation of state variable in (2) is modified as

$$
\hat{x}\left(k_{i}+1\right)=A \hat{x}\left(k_{i}\right)+B \Delta u\left(k_{i}\right)+K_{o b}\left(y\left(k_{i}\right)-C \hat{x}\left(k_{i}\right)\right) .
$$

The cost function and optimal solution are similar to that of (5) and (6), respectively, except that the term $x(\cdot)$ is replaced by $\hat{x}(\cdot)$.

We need to modify the above solution if operational constraints are introduced to the model. The constraints are:

(1) Constraints on rate of change of all the control variables within the future control trajectory started from timestep $k_{i}$ :

$$
\Delta U^{\min } \leq \Delta U \leq \Delta U^{\max } .
$$

(2) Constraints on amplitude of all the control variables within the future control trajectory:

$$
U^{\min } \leq U \leq U^{\max }
$$

(3) Constraints on the outputs within the prediction trajectory:

$$
Y^{\min } \leq Y \leq Y^{\max }
$$

The constraints in (10) can be rewritten in a matrix form:

$$
\left[\begin{array}{c}
-I \\
I
\end{array}\right] \Delta U \leq\left[\begin{array}{c}
-\Delta U^{\min } \\
\Delta U^{\max }
\end{array}\right]
$$

In (11), $U=\left[\begin{array}{llll}u\left(k_{i}\right)^{T} & u\left(k_{i}+1\right)^{T} & \cdots & u\left(k_{i}+N_{c}-1\right)^{T}\end{array}\right]^{T}$, which can be expressed as

$$
U=\underbrace{\left[\begin{array}{c}
I \\
\vdots \\
I
\end{array}\right]}_{C_{1}} u\left(k_{i}-1\right)+\underbrace{\left[\begin{array}{cccc}
I & O & \cdots & O \\
\vdots & \vdots & \ddots & \vdots \\
I & I & \cdots & I
\end{array}\right]}_{C_{2}} \underbrace{\left[\begin{array}{c}
\Delta u\left(k_{i}\right) \\
\vdots \\
\Delta u\left(k_{i}+N_{c}-1\right)
\end{array}\right]}_{\Delta U},
$$

where $I$ and $O$ are the identity and zero matrices with dimension of $m \times m$. From (14), we can represent (11) as

$$
\left[\begin{array}{c}
-C_{2} \\
C_{2}
\end{array}\right] \Delta U \leq\left[\begin{array}{c}
-U^{\min }+C_{1} u\left(k_{i}-1\right) \\
U^{\max }-C_{1} u\left(k_{i}-1\right)
\end{array}\right] .
$$

From (4), the output constraints can be rewritten as

$$
\left[\begin{array}{c}
-\Phi \\
\Phi
\end{array}\right] \Delta U \leq\left[\begin{array}{c}
-Y^{\min }+F x\left(k_{i}\right) \\
Y^{\max }-F x\left(k_{i}\right)
\end{array}\right]
$$

Finally, solving the MPC with constraints is to find $\Delta U$ that minimizes the cost function in (5) subject to the inequality constraints in (13), (15), and (16). This is a quadratic programming problem with linear inequality constraints:

$$
\begin{aligned}
& J=\frac{1}{2} x^{T} E x+x^{T} F, \\
& M x \leq \gamma,
\end{aligned}
$$

where $E$ and $F$ can be inferred from (5); $M$ and $\gamma$ are a matrix and a vector reflecting the constraints.

The optimization problem in (17) can be solved using various methods. This work uses a primal-dual interior-point algorithm [2] employing the predictor-corrector method [3] which is computational and resource efficient. 


\section{Minimal Model}

There are many mathematical blood glucose models proposed in the literature [4]. Among them, the minimal model proposed by Bergman et al. (1981) [5] is the most popular model due to its simplicity and physiological accuracy. This work uses the Fisher model [6], a modified version of the minimal model, for the regulation of blood glucose using MPC. The model equations are

$$
\begin{aligned}
\dot{G} & =-p_{1} G-X\left(G+G_{B}\right)+P(t), \\
\dot{X} & =-p_{2} X+p_{3} I, \\
\dot{I} & =-n\left(I+I_{B}\right)+u(t) / V_{I} .
\end{aligned}
$$

In (18), $G(t)$ and $I(t)$, respectively, are the differences of plasma glucose concentration and free plasma insulin concentration from their basal values which are $G_{B}$ and $I_{B}$, respectively; $X(t)$ is proportional to the concentration of insulin in the remote compartment; $P(t)$ and $u(t)$ are the rates of infusion of exogeneous glucose and insulin, respectively; $V_{I}$ is the insulin distribution volume and $n$ is fractional disappearance rate of insulin.

The following discrete model is obtained by linearizing the model with the steady-state values of $G=I=X=P=0$ :

$$
\begin{aligned}
x_{k+1} & =A x_{k}+B u_{k}+D d_{k}, \\
y_{k} & =C x_{k},
\end{aligned}
$$

where $x, u$ and $y$ represent the state variable, input and output of the system, respectively; $k$ is the discrete time-step.

With the model parameters adopted from [6]: $p_{1}=0, p_{2}=$ $0.025, p_{3}=0.000013, V_{I}=12, n=5 / 54, G_{B}=4.5, I_{B}=15$, and the sampling time of 5 minutes, the parameter matrices in (19) are:

$$
\begin{gathered}
A=\left[\begin{array}{ccc}
1 & -2.115 \times 10^{1} & -6.039 \times 10^{-4} \\
0 & 8.825 \times 10^{-1} & 4.867 \times 10^{-5} \\
0 & 0 & 6.294 \times 10^{-1}
\end{array}\right], C=\left[\begin{array}{lll}
1 & 0 & 0
\end{array}\right], \\
B=\left[\begin{array}{c}
-8.799 \times 10^{-5} \\
1.118 \times 10^{-5} \\
3.335 \times 10^{-1}
\end{array}\right], D=\left[\begin{array}{lll}
5 & 0 & 0
\end{array}\right]^{T} .
\end{gathered}
$$

The term $P(t)$ in (18) represents the rate of glucose entering the blood from intestinal absorption after a meal, and can be estimated by the following function [7]:

$$
P(t)=\frac{D_{G} A_{G} t e^{-t / t_{\max , G}}}{t_{\max , G}^{2}},
$$

where $D_{G}$ is the amount of carbohydrates digested, $A_{G}$ represents the carbohydrate bioavailability, and $t_{\max , G}$ is the time from the beginning of the meal consumption until the absorption rate reaches its maximum. $A_{G}$ and $t_{\max , G}$ are chosen as 0.8 and 40 minutes, respectively, as in [7]. Hence, the term $d_{k}$ in (19) is determined as

$$
d_{k}=\left\{\begin{array}{cc}
0.0025 D_{G}\left(k-k_{G}\right) e^{-0.125\left(k-k_{G}\right)} & \text { if } k \geq k_{G}, \\
0 & \text { otherwise, }
\end{array}\right.
$$

where $k_{G}$ is the time-step at which the meal disturbance is implemented.

The set-point is chosen as $5.5 \mathrm{mmol} / \mathrm{L}$ in our experiments. In addition, due to limitations on the range and possible changes of insulin infusion rate that can be enforced by a common insulin pump, and conditions of the output to avoid hypoglycemia and extreme hyperglycemia, the following constraints are also imposed [8]:

$$
\begin{array}{r}
0 \leq u \leq 80 \mathrm{mU} / \mathrm{min}, \\
-16.7 \leq \Delta u \leq 16.7 \mathrm{mU} / \mathrm{min}, \\
3 \leq y \leq 15 \mathrm{mmol} / \mathrm{L} .
\end{array}
$$

Since measurements of $X$ are physically unavailable, and those of $I$ are inaccessible in practice, we need to estimate the state variable $x$ using an observer. Hence, the optimization problem is listed in (17), where all the parameter matrices can be easily inferred from (5), (9), (19), (21), and (22).

\section{RESUlTS AND DisCUSSION}

We implemented the proposed MPC design and other controller modules for glucose regulation on a microcontroller. Since our aim is to develop a real-time implantable close-loop glucose control system, the hardware components should be bio-compatible, light weight, small in size, and energy efficient. The system should comprise a microprocessor that has sufficient computational power to perform the necessary calculation in real-time. Based on these criterions and an empirical competitive study of different microcontrollers [9], Texas Instruments (TI) MSP430F5438A, a 16-bit ultra-low-power RISC microcontroller with 256KB Flash and 16KB SRAM, was chosen as the processor. Other components include the TI very-low-power RF transceiver CC1101, a small-in-size Mitsubishi chip antenna, the Bartels mp6 micropump, an insulin reservoir, and a power-supply and charging unit.

Figure 1 shows an overview of our system. In operation mode, the plasma glucose level is measured at an interval of every 5 minutes by the glucose sensor. This value is transmitted to the processor which executes the MPC algorithm to compute the insulin to be injected into the blood vessel through the micropump. The processor can send the operating status to a base station and receive operation commands and patient parameters from the base station through wireless communications. Both the artificial pancreas and the base station can be connected through serial communications to a computer for debugging, data analyzing, and patient management. 


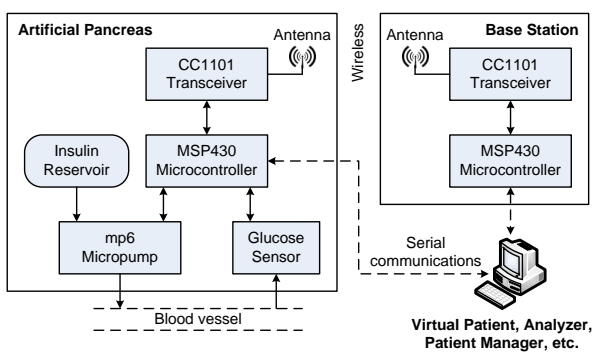

(a)

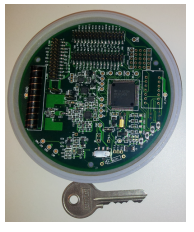

(b)

Fig. 1: Overview of the system : (a) Block diagram; (b) Printed circuit board of the artificial pancreas.

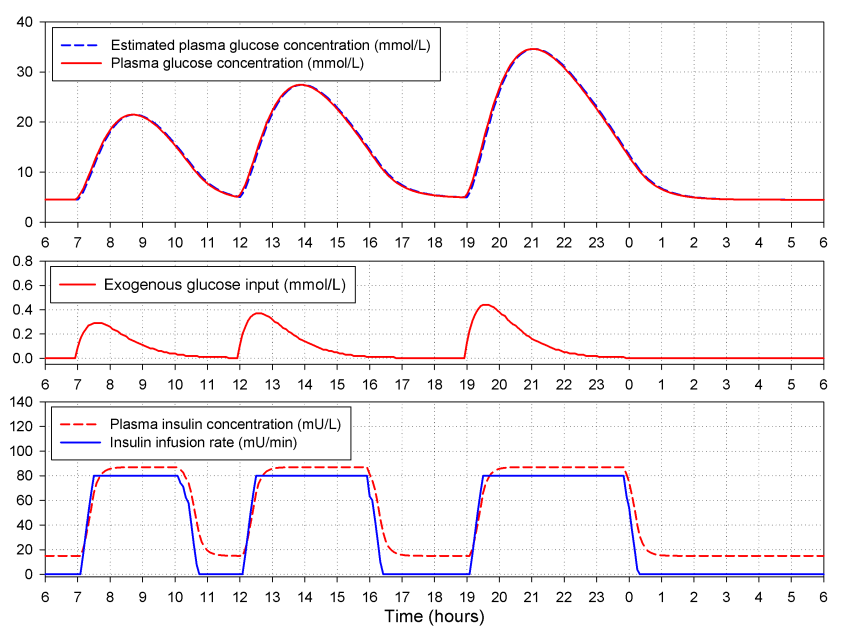

Fig. 2: Artificial Pancreas - Virtual Patient simulation results.

A Virtual Patient using the minimal model was implemented on the computer to verify the accuracy of the MPC algorithm embedded within the artificial pancreas. Figure 2 shows the simulation results for a patient who has breakfast, lunch, and dinner at 7:00, 12:00, and 19:00, respectively. The corresponding meal sizes are $50 \mathrm{~g}, 60 \mathrm{~g}$, and $70 \mathrm{~g}$ of carbohydrates, respectively. As seen in Figure 2, the patient blood glucose reach the maximum level after the meal starts about 2 hours. However, with a prediction window of 400 minutes $\left(N_{P}=80\right)$ and appropriate meal size, the MPC-based artificial pancreas is able to bring down the glucose level to $5 \mathrm{mmol} / \mathrm{L}$ 5-7 hours after the meal.

The binary code of the MPC and serial communications on the MSP430F5438A microcontroller is $23 \mathrm{~KB}$, which is smaller than that of other implementations $[10,11]$. The time for computation and communications during each time-step was less than 1 second on average. With longer prediction and control windows $\left(N_{P}=80, N_{C}=4\right)$, the computation

speed of our system is comparable with that of the other designs $[10,11]$. In addition, this system is highly energy efficient since most of the time the processor is on standby mode with the power consumption rate of $2.1 \mu \mathrm{A}$ at $3.0 \mathrm{~V}$. The power consumption rate in active mode is about $230 \mu \mathrm{A} / \mathrm{MHz}$ at $8 \mathrm{MHz}, 3.0 \mathrm{~V}$. The microcontroller takes less than $5 \mu \mathrm{s}$ to be active from standby mode.

\section{CONCLUSION}

We have proposed an implementation of MPC with constraints on a microcontroller, and apply this implementation on the development of a real-time implantable close-loop glucose control system. The experimental results show that our artificial pancreas has low power consumption and a reasonable small size, and the chosen microprocessor is capable of performing the computational efficient MPC algorithm accurately and in real-time. This prototype device will be tested in an animal experiment to be conducted this year.

\section{ACKNOWLEDGEMENTS}

This work is supported in parts by a research grant from the BEP program of Agency of Science, Technology and Research (A*STAR), Singapore (Grant No. 1021480003).

\section{REFERENCES}

1. Wu Z, Chui C-K, Hong G-S, Chang S. Physiological analysis on oscillatory behavior of glucose-insulin regulation by model with delays $J$ Theor Biol. 2010;280:1-9.

2. Mehrotra S. On the implementation of a primal-dual interior point method SIAM J Optim. 1992;2:575-601.

3. Wright S J. Primal-dual interior-point methods. SIAM 1997.

4. Balakrishnan N P, Rangaiah G P, Samavedham L. Review and analysis of blood glucose (BG) models for type 1 diabetic patients Ind. Eng. Chem. Res. 2011;50:12041-12066.

5. Bergman R N, Phillips L S, Cobelli C. Physiologic evaluation of factors controlling glucose tolerance in man: measurement of insulin sensitivity and beta-cell glucose sensitivity from the response to intravenous glucose J. Clin. Invest. 1981;68:1456-1467.

6. Fisher M E. A semiclosed-loop algorithm for the control of blood glucose levels in diabetics IEEE Trans. Biomed. Eng. 1991;38:57-61.

7. Hovorka R, Canonico V, Chassin L J, et al. Nonlinear model predictive control of glucose concentration in subjects with type 1 diabetes Physiol Meas. 2004;25:905-920.

8. Lynch S M, Bequette B W. Estimation-based model predictive control of blood glucose in type I diabetics: a simulation study in Proc. NEBC 2001:79-80 2001.

9. Goh W, Venkat K. MSP430 competitive benchmarking 2006

10. Bleris L G, Kothare M V. Real-time implementation of model predictive control in Proc. ACC 2005;6:4166-4171 2005.

11. Bleris L G, Kothare M V. Implementation of model predictive control for glucose regulation on a general purpose microprocessor in Proc. CDC-ECC 2005:5162-5167 2005. 